\title{
Trajectory of a synthetic jet issuing into high Reynolds number turbulent boundary layers
}

\author{
Tim Berk ${ }^{1}$, Nicholas Hutchins ${ }^{2}$, Ivan Marusic ${ }^{2}$ \\ and Bharathram Ganapathisubramani ${ }^{1} \dagger$ \\ ${ }^{1}$ Aerodynamics and Flight Mechanics Research Group, University of Southampton, \\ Southampton SO17 1BJ, UK \\ ${ }^{2}$ Department of Mechanical Engineering, The University of Melbourne, Victoria 3010, \\ Australia
}

(Received $\mathrm{xx}$; revised $\mathrm{xx}$; accepted $\mathrm{xx}$ )

Synthetic jets are zero-net-mass-flux actuators that can be used in a range of flow control applications. For some applications the scaling of the trajectory of the jet with actuation and cross-flow parameters is important. This scaling is investigated for changes in the friction Reynolds number, changes in the velocity ratio (defined as a ratio between the mean jet blowing velocity and the free-stream velocity) and changes in the actuation frequency of the jet. A distinctive aspect of this study are the high-Reynolds number turbulent boundary layers (up to $R e_{\tau}=12800$ ) of the cross flow. To our knowledge, this is the first study to investigate the effect of the friction Reynolds number of the cross-flow on the trajectory of an (unsteady) jet, as well as the first study to systematically investigate the scaling of the trajectory with actuation frequency. A broad range of parameters is varied (rather than an in-depth investigation of a single parameter) and the results of this study are meant to indicate the relative importance of each parameter rather than the exact influence on the trajectory. Within the range of parameters explored, the critical ones are found to be the velocity ratio as well as a non-dimensional frequency based on the jet actuation frequency, the cross-flow velocity and the jet dimensions. The Reynolds number of the boundary layer is shown to only have a small effect on the trajectory. An expression for the trajectory of the jet is derived from the data, which (in the limit) is consistent with known expressions for the trajectory of a steady jet in a cross-flow.

\section{Introduction}

The trajectory of a steady jet in cross flow has been widely studied in the literature (see for an overview reviews by Margason 1993 and Mahesh 2013). When the jet is forced or pulsed at a fixed frequency its trajectory can be altered significantly (Vermeulen et al. 1990). While the scaling of the trajectory with jet velocity is fairly well established, the scaling of trajectory with frequency has not been studied in detail in the literature. Three cases are considered here in which a jet in cross flow is subjected to a frequency component: a forced jet in which a periodic variation is imposed on a non-zero mean jet velocity, a pulsed jet in which discrete pulses of the jet are alternated with zero velocity between pulses and a synthetic jet in which equal blowing and suction pulses are alternated leading to a zero-net-mass-flux flow at the jet exit but a train of blowing pulses away from the jet exit. In the following these three cases are generalized using the term unsteady jet. The velocity cycle of such an unsteady jet can be generalized as $u_{j}(t)=\left\langle u_{j}\right\rangle+u_{j}^{\prime}(t / T)$, where $\left\langle u_{j}\right\rangle$ represents the time-averaged jet velocity and $u_{j}^{\prime}$ the

$\dagger$ Email address for correspondence: G.Bharath@soton.ac.uk 
cyclic fluctuations around this average with frequency $f=1 / T$. When using sinusoidal forcing as in the present study, the velocity cycle is described by

$$
u_{j}(t)=\left\langle u_{j}\right\rangle+u_{j}^{\prime} \sin (2 \pi t f) .
$$

The forced jet is the most general case with usually $\left\langle u_{j}\right\rangle>u_{j}^{\prime}$ while pulsed and synthetic jets are special cases where $\left\langle u_{j}\right\rangle=u_{j}^{\prime}$ for pulsed jets, and $\left\langle u_{j}\right\rangle=0$ for synthetic jets. (Note that forced and pulsed jets are generally driven by a square wave instead of a sinusoidal wave.) These three cases have some clear differences (especially between blowing pulses, where $u_{j}>0$ for forced jets, $u_{j}=0$ for pulsed jets and $u_{j}<0$ for synthetic jets) but also have significant similarity in that coherent vortical structures are formed with the specified frequency (see for example Wu et al. (1988) for forced jets, Eroglu and Breidenthal (2001) for pulsed jets and Jabbal and Zhong (2008) for synthetic jets). It has been suggested that variations in the trajectory are related to the distance between these vortical structures (Eroglu and Breidenthal 2001; Jabbal and Zhong 2010). If so, scaling of the trajectory with frequency might be analogous between the three unsteady jet cases. The present study focusses on deriving this scaling by fitting empirical data of a synthetic jet in a turbulent boundary layer.

Applications of unsteady jets in cross flow include control of the jet in cross flow (Narayanan et al. 2003), separation control (Dandois et al. 2007), thrust vector control (Miller et al. 2001), film cooling (Muldoon and Acharya 2009) and skin-friction control. Recent results have shown that skin-friction drag can be reduced up to $3 \%$ by targeting the large-scale structures of a high-Reynolds number turbulent boundary layer using a pulsed jet (Abbassi et al. 2017). In similar experiments, the near-wall structures of a turbulent boundary layer were targeted using a synthetic jet (Rathnasingham and Breuer 2003). A common feature in these studies is the rectangular orifice shape flush with the wall and aligned with the long dimension in streamwise direction, analogous to the orientation of near-wall streaks and large-scale structures in the turbulent boundary layer. In these control studies incoming structures in the flow are detected upstream of the orifice, upon which the jet is fired to target these structures. For the efficient targeting of structures away from the wall it is important to know the trajectory of the jet. The present study is motivated by the application of skin-friction control, which is reflected in the rectangular orifice geometry and the low ratio of jet velocity to cross-flow velocity compared to some other studies (this is required for the jet to stay inside the boundary layer).

In addition to the trajectory of a jet, spread (Davitian et al. 2010), mixing (Smith and Mungal 1998), entrainment (Narayanan et al. 2003), bifurcation (Eroglu and Breidenthal 2001) and shear-layer instabilities (Megerian et al. 2007) are important factors in the development of a jet in cross flow (Mahesh 2013; Karagozian 2014). Given the stated motivation of targeting structures in the flow, the present study focuses on the trajectory only. Note that bifurcation of the jet has not been observed in the present study and the trajectory consists of a single branch.

The remainder of this introduction starts with a description of the canonical case: a steady circular jet issuing in a uniform cross flow. This is followed by a discussion on the effect of an actuation frequency (i.e. an unsteady jet). Next, the influence of changing the orifice shape (rectangular instead of circular) is examined and some considerations for an orifice length scale are presented. Then, the influence of issuing into turbulent boundary layers (as opposed to laminar boundary layers or a uniform cross flow) is discussed. Finally, the important parameters are summarized and scaling parameters are proposed that describe the influence of the mentioned aspects on the trajectory. 


\subsection{Canonical case: steady circular jet}

The canonical case for the jet in cross flow is a steady circular jet in a uniform cross flow (Johari 2006; Mahesh 2013). Jet trajectories are typically normalized by a relevant orifice length-scale $D$ (usually the diameter for circular jets) and the velocity ratio between the jet and the free-stream velocities, $r=u_{j} / U_{\infty}$. For example, Broadwell and Breidenthal (1984) derived a scaling $r D$ based on the jet as point-source of momentum, while Karagozian (1986) derived a scaling including viscous effects which can be expressed as $r^{1.7} D$ and Keffer and Baines (1962) found a collapse of their data for $r^{2} D$. Using a generalized scaling of $r^{c} D$, the trajectory of a jet in cross flow is usually described by an empirical power-law of the form

$$
\frac{y}{r^{c} D}=B\left(\frac{x}{r^{c} D}\right)^{n}
$$

where $y$ is the wall-normal location, $x$ is the streamwise location and $B, n$ and $c$ are constants. A comparison by Mahesh (2013), considering different studies, shows that trajectories do not fully collapse when normalized by $r D$, indicating that $B, n$ and $c$ are not constant. Indeed, a range is reported of $1.2<B<2.6,0.28<n<0.34$ and $0<c<2$ (Mahesh 2013). Although most authors define the trajectory using a single scaling, Smith and Mungal (1998) defined three regions of the transverse jet, each with a different length scale: a vortex interaction region just downstream of the orifice scaling with $D$, a near-field scaling with $r D$ and a far-field scaling with $r^{2} D$. Broadwell and Breidenthal (1984) defined a far field as the region where structures created by the jet move with the cross-flow velocity, limited by $x \gg r \sqrt{\pi D^{2} / 4}$. Using this definition, the low velocity ratios used in the present study cause all measurements to be performed in the far field.

The development of a jet in cross flow can be (inadequately) described in terms of momentum fluxes by decomposing the trajectory into a wall-normal and a streamwise part. The wall-normal development (or penetration) of the jet is dominated by the momentum flux created by the jet at the orifice. As the jet penetrates into the cross flow, the initial wall-normal momentum decreases due to drag. At the same time, the cross flow transfers streamwise momentum to the jet, accelerating the jet until the crossflow velocity is reached. This combination causes the trajectory to evolve from pointing in the wall-normal direction at the orifice to pointing in the streamwise direction in the far-field.

The above analysis leads to an incomplete description of the jet since the interaction with the cross flow leads to a complex set of coherent vortical structures (Fric and Roshko 1994). The cross section of a jet in cross flow is dominated by a counter-rotating vortex pair (e.g. Karagozian 2014). This vortex pair creates a self-induced velocity on the jet in the vertical direction, influencing the trajectory. In the far-field where the initial wallnormal momentum has decayed, the velocity induced by this vortex pair is shown to drive the wall-normal penetration (Broadwell and Breidenthal 1984). In fact, multiple theoretical descriptions of the trajectory have been derived based on the strength and induced velocity of this counter-rotating vortex pair (e.g. Broadwell and Breidenthal 1984 or Karagozian 1986).

\subsection{Unsteady jets}

It may be assumed that for unsteady jets the velocity induced by created vortical structures has an equally important effect on the trajectory. Johari (2006) derived scaling parameters for the penetration of isolated vortex rings into a cross flow. These parameters are based on the impulse of the vortex ring (directly related to the circulation) and 
ultimately lead to a scaling of the trajectory with the non-dimensional stroke length which represents the length of the slug of fluid ejected each pulse and is related to the optimal formation of vortex rings (Gharib et al. 1998). However, in most cases the interaction between an unsteady jet and a cross flow does not produce isolated vortex rings but a train of non-trivial vortical structures that interact with each other. Jabbal and Zhong (2010) showed that the trajectory of synthetic jets in a cross flow depends on the type of vortical structures created by the jet. Depending on the velocity ratio and non-dimensional frequency, the interaction of a pulsed or synthetic jet with a cross flow can lead to vortical structures such as tilted, distorted and stretched vortex rings or hairpin vortices (Jabbal and Zhong 2008; Sau and Mahesh 2008). The velocity induced by a single vortical structure depends on the circulation and the geometry of the structure. In addition, for a train of vortical structures as created by a forced, pulsed or synthetic jet, the spacing and interaction between vortical structures also play an important role (Eroglu and Breidenthal 2001; Jabbal and Zhong 2010). Due to the interaction with the cross-flow boundary layer, the circulation (partially cancelled by the shear in the cross flow) and geometry of these structures are non-trivial functions of the actuation and crossflow parameters. This implies that theoretical scalings for the trajectory such as derived by Broadwell and Breidenthal (1984) and Karagozian (1986) for a counter-rotating vortex pair or by Johari (2006) for isolated vortex rings would be highly dependent on these parameters for an unsteady jet in a cross flow. Therefore, theoretical scaling laws are hard to formulate when such geometrically complicated vortical structures are formed. The present work does not consider the exact mechanisms in which the actuation and cross-flow parameters influence the trajectory, but rather investigates the variation and scaling of the trajectory with these parameters.

The trajectory of an unsteady jet is generally described using equation 1.2 (e.g. Eroglu and Breidenthal 2001; Smith 2002), which is independent of actuation frequency. However, as discussed above and shown in numerous studies (e.g. Vermeulen et al. 1990; Johari et al. 1999; Eroglu and Breidenthal 2001; M'Closkey et al. 2002; Shuster et al. 2005; Johari 2006; Shapiro et al. 2006) the frequency of an unsteady jet plays an important role in the trajectory through the geometry of the created structures, the circulation of each structure and the distance between subsequent structures. It has to be noted that some studies argue that the duty cycle and pulse width (for square-wave excitation of a forced jet) are more important parameters than the actuation frequency (Shapiro et al. 2006). Associated time scales are linked to the non-dimensional stroke length for vortex-ring formation (Gharib et al. 1998) and to shear-layer instabilities (see the recent review by Karagozian 2014). For the present study on synthetic jets with a sinusoidal waveform, the duty cycle is fixed to 0.5 and the pulse width is a direct function of the frequency. Care should be taken when comparing the results of the present study to studies using square-wave forcing, especially when duty cycles other than 0.5 are considered.

In addition to the direct effect of frequency, an important aspect of an unsteady jet is the fluctuating velocity ratio $u_{j}(t) / U_{\infty}$. Usually a single velocity magnitude is defined for the jet, which can be based on the time-averaged jet velocity $\left\langle u_{j}(t)\right\rangle$ (e.g. Karagozian 2014), time-averaged momentum flow velocity $U_{0}$ (e.g. Eroglu and Breidenthal 2001; Cater and Soria 2002) or mean velocity of the blowing part of the cycle $\bar{u}$ (e.g. Johari 2006; Smith and Glezer 1998). For a sinusoidal velocity cycle with $\left\langle u_{j}(t)\right\rangle=0$ (i.e. a synthetic jet) and a constant orifice area the momentum flow velocity and mean blowing velocity are given by $U_{0}=u_{j}^{\prime} / \sqrt{2}$ and $\bar{u}=u_{j}^{\prime} / \pi$ respectively (with $u_{j}^{\prime}$ as defined in equation 1.1), i.e. $U_{0}=\bar{u} \pi / \sqrt{2}$. Following a large body of literature on synthetic jets, the present study characterizes the jet using the mean blowing velocity (Smith and Glezer 
1998). Using this velocity magnitude, the waveform of a sinusoidal synthetic jet is fully described by the velocity magnitude $\bar{u}$ and frequency $f$. The velocity ratio is then defined as

$$
r=\bar{u} / U_{\infty}
$$

It should be noted that the comparison to a steady jet and other types of unsteady jets might be more appropriate based on the time-averaged momentum of the jet $U_{0}$ (Van Buren and Amitay 2016). Any discrepancy is expected to appear in the constant $B$ in equation 1.2 and $A$ in equation 1.6. Neither of these constants influence the scaling investigated in the present study.

\subsection{Orifice length scale}

In addition to the discussed complicated effect of frequency, the present study differs from the canonical case by using a rectangular orifice shape. This orifice has a constant length $l$ and width $d$ and is described by the aspect ratio,

$$
A R=l / d .
$$

The relevant orifice length scale $D$ as appearing in equation 1.2, which is usually taken to be the diameter of a circular orifice, now needs to be based on the dimensions of a rectangular orifice. Broadwell and Breidenthal (1984) treated the jet as a point-source of momentum, leading to a length scale equal to the square-root of the cross-sectional area of the orifice, which is proportional to the diameter for a circular orifice and is given by $(l d)^{1 / 2}$ for a rectangular orifice. O'Farrell and Dabiri (2014) showed that the equivalent diameter, also scaling with $(l d)^{1 / 2}$, is the relevant length scale for formation of vortex rings from rectangular orifices in quiescent flow. These arguments suggest an orifice length scale of $D=(l d)^{1 / 2}$. However, Van Buren et al. (2016) showed an influence of the orientation of a rectangular orifice compared to the flow. A synthetic jet actuator with a rectangular orifice of aspect ratio $A R=18$ was placed in a cross flow with the long dimension of the slot either aligned in spanwise- or in streamwise direction. They show that this has a significant effect on both the type of structures created by the jet and on the trajectory, ruling out a length scale of $(l d)^{1 / 2}$ which is independent of orientation. The discrepancy with the scaling derived by Broadwell and Breidenthal (1984) can be explained by the deviation from a point-source of momentum when placing a rectangular orifice with the long dimension perpendicular to the cross flow. This will cause the cross flow to impact on a relatively large surface of the jet, which is not accounted for by the point-source approach.

Another candidate for the relevant orifice length scale could be the length of the slot in streamwise direction. However, this dimension is independent of the aspect ratio of the slot, which has been shown to be an important parameter for vortex formation (Van Buren et al. 2014). Moreover, the streamwise length $l$ of a slot is the same as the streamwise length of a circular orifice with diameter $l$, while these have been shown to have a very different influence on the flow field (Kim et al. 2012). The same arguments rule out the length of the slot in spanwise direction as the relevant length scale.

The relevant orifice length scale for scaling of the trajectory using a rectangular orifice is not explored in the literature. While being equal to the diameter for a circular orifice, for a rectangular orifice this length scale depends on the slot width, slot length and orientation compared to the cross flow. The present study uses a fixed orifice geometry and orientation (based on the skin-friction control motivation discussed above) which does not give any insight in the nature of this relevant length scale. However, it may be assumed that the constant orifice geometry leads to a constant length scale $D$ and 
the results for scaling of the actuation frequency are assumed to be independent of this length scale. The nature of $D$ is outside the scope of this current study and we use an arbitrary value of $D=1 \mathrm{~m}$ where quantification is required.

\subsection{Turbulent boundary layer}

The present study investigates the trajectory of a rectangular synthetic jet issuing into different turbulent boundary layers. The turbulent boundary layers are characterized using the free-stream velocity $U_{\infty}$, the boundary-layer thickness $\delta$, the kinematic viscosity $\nu$ and the skin-friction velocity $U_{\tau}$. It should be noted that the friction velocity is directly related to the free-stream velocity through the skin-friction coefficient $c_{f}=2 U_{\tau}^{2} / U_{\infty}^{2}$, which is a function of $\log \left(R e_{x}\right)$. For the high values of $R e_{x}$ used in the present study (required for high-Reynolds number turbulent boundary layers) $\log \left(R e_{x}\right)$ is relatively constant, leading to a fixed ratio between $U_{\tau}$ and $U_{\infty}$. Therefore, the free-stream velocity and friction velocity are not varied independently in the present study. The governing non-dimensional parameter describing a turbulent boundary layer is the friction Reynolds number (e.g. Klewicki 2010; Marusic et al. 2010; Smits et al. 2011), defined by

$$
R e_{\tau}=U_{\tau} \delta / \nu
$$

This Reynolds number can be interpreted as the ratio between the two characteristic length scales in a turbulent boundary layer: the inner length-scale $\nu / U_{\tau}$ and the outer length-scale $\delta$.

Muppidi and Mahesh (2005) studied the influence of the boundary-layer thickness on the trajectory of a steady jet. The main influence identified by these authors was the wall-normal variation of cross-flow velocity in the boundary layer, leading to different (local) values of the velocity ratio.

An additional influence of the turbulent boundary layer is the distribution of vorticity in the cross flow. It has been shown that the vorticity in the boundary layer (partially) cancels the vorticity in the upstream side of vortical structures created by a pulsed jet (Sau and Mahesh 2008). Lim et al. (2008) showed that the tilt of vortex rings in a cross flow depends on the shear in the cross flow. These studies suggest that the shape of the boundary layer influences the geometry and behaviour of vortical structures, thereby influencing the trajectory of the jet.

\subsection{Parameter space and scaling law}

Summarizing all the parameters discussed above, the synthetic jet is described by the velocity magnitude $\bar{u}$, frequency $f$ and (unknown but constant) characteristic length scale $D$ while the cross flow is described by the free-stream velocity $U_{\infty}$, boundary-layer thickness $\delta$, friction velocity $U_{\tau}$ and kinematic viscosity $\nu$.

Analogous to a steady jet in cross flow as discussed above, it is hypothesised that the trajectory of a synthetic jet can also be described by a power law of the form

$$
\frac{y}{g(\ldots) D}=A\left(\frac{x}{g(\ldots) D}\right)^{n},
$$

where $g(\ldots)$ represents a non-dimensional scaling factor depending on jet and cross-flow parameters. Note that this is a very general expression which is equivalent to power-laws of the form $y / D=A h(\ldots)(x / D)^{n}$ as found in for example Karagozian (1986) or Johari (2006). The validity of assuming a power-law for the trajectory is discussed in $\S 3.1$.

For steady jets in a laminar cross flow (equation 1.2) $g(\ldots)$ equals the velocity ratio 
to the power $c$,

$$
g(\ldots)=r^{c}=\left(\bar{u} / U_{\infty}\right)^{c} .
$$

It is hypothesized that issuing a jet into a turbulent boundary layer (as opposed to a laminar cross flow in most studies) will add an influence of $R e_{\tau}$ on $g(\ldots)$. As discussed above, issuing a synthetic jet instead of a steady jet into a cross flow will also add an influence of the frequency. The correct non-dimensional form for this frequency is unknown. Three scalings are hypothesized for this frequency. These are the outer- and inner units of the boundary layer or a passing frequency of the cross flow over the jet, i.e.

$$
\begin{aligned}
f_{1}^{*} & =f \delta / U_{\infty}, \\
f_{2}^{*} & =f \nu / U_{\tau}^{2}, \\
f_{3}^{*} & =f D / U_{\infty} .
\end{aligned}
$$

The influence of the velocity ratio, the friction Reynolds number and the nondimensional frequency on the trajectory is combined by assuming a scaling for unsteady jets of

$$
g(\ldots)=r^{c_{1}} \operatorname{Re}_{\tau}^{c_{2}} f^{* c_{3}} .
$$

To identify the suitable non-dimensional frequency, the constants $c_{1}, c_{2}$ and $c_{3}$ are fitted against the obtained data for $f_{1}^{*}, f_{2}^{*}$ and $f_{3}^{*}$ independently and residual errors are compared against each other. The validity of the assumed relevant non-dimensional groups is discussed in appendix A by fitting the data against physical parameters first (i.e. $g(\ldots)=\bar{u}^{\alpha_{1}} f^{\alpha_{2}} D^{\alpha_{3}} U_{\infty}^{\alpha_{4}} \delta^{\alpha_{5}} \nu^{\alpha_{6}}$ ) after which non-dimensional groups are formed from these parameters, leading to the same groups as in equation 1.11.

The purpose of the present study is to identify the scaling of the trajectory as in equation 1.6, including the suitable non-dimensional jet frequency for the interaction of a synthetic jet with a high-Reynolds number turbulent boundary layer. Compared to the literature, the present study is performed using very high friction Reynolds numbers. Velocity ratios used in this study are low compared to those found in most other studies. The number of cases is limited, meaning that the fitted coefficients have a significant uncertainty. The goal is not to find the exact values of these coefficients but rather to study the relative importance of different parameters, using the broad range of parameters varied in this study.

\section{Experimental set-up and procedures}

\subsection{Description of the actuator}

A synthetic-jet actuator is located underneath the wind tunnel floor, with the nozzle flush with the floor and the jet perpendicular to both the floor and the cross flow at the jet exit. The actuator is driven by a Visaton SC $8 \mathrm{~N}$ loudspeaker, actuated with a sinusoidal velocity signal. This speaker has a frequency response of 70-20000 Hz with a resonance frequency around $90 \mathrm{~Hz}$. This speaker is attached to a circular (pancake shaped) cavity of volume $1.85 \times 10^{-5} \mathrm{~m}^{3}$ with the $6.8 \times 10^{-2} \mathrm{~m}$ diameter aligned in spanwise-wallnormal direction and the $5 \times 10^{-3} \mathrm{~m}$ height aligned in streamwise direction relative to the wind tunnel. The cavity is attached to a nozzle with a neck length of $19.5 \mathrm{~mm}$, slot length of $l=13 \mathrm{~mm}$ and width of $d=1 \mathrm{~mm}(A R=13)$. In absence of a cross flow, this combination of cavity and nozzle leads to a fluidic resonance frequency of $550 \mathrm{~Hz}$ as determined from exit velocity measurements. The long-dimension of the slot $(l)$ is aligned 


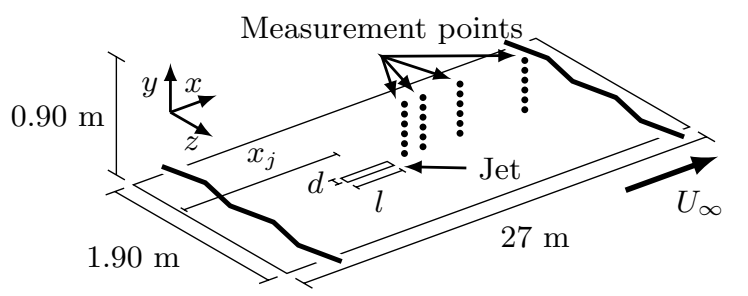

FIgURE 1. Schematic of set-up, indicating slot position and orientation as well as measurement locations and relevant parameters (not to scale).

with the flow as presented schematically in figure 1. Details on the jet calibration are given in $\S 2.3$.

\subsection{Description of the facility}

Experiments are performed in the high-Reynolds number boundary-layer wind tunnel (HRNBLWT) at the University of Melbourne. This open-return blower wind tunnel has a $27 \mathrm{~m}$ long test section, enabling Reynolds numbers up to $R e_{\tau}=25000$. The test section has a spanwise width of $1.9 \mathrm{~m}$ and wall-normal height of $0.9 \mathrm{~m}$, meaning that the influence of the side-walls and ceiling on the trajectory of the jet can be neglected. See figure 1 for a schematic of the set-up. The local boundary-layer thickness is a function of both the free-stream velocity and the downstream distance from the boundary-layer trip at the test-section inlet. By varying the downstream location of the actuator $\left(x_{j}\right)$, the boundary-layer thickness can be varied independently from the free-stream velocity. The friction velocity $U_{\tau}$ cannot be controlled independently and is a function of mainly the free-stream velocity $\left(U_{\infty}\right)$ and streamwise location $\left(x_{j}\right)$. Experimental parameters were intentionally varied from case to case and are listed in $\S 3$. Further details on the facility can be found in Nickels et al. (2005).

Velocity measurements are performed using a single-wire traversing hot-wire probe, operated in constant-temperature mode with an overheat ratio of 1.7. The physical length of the wire was kept constant at $0.5 \mathrm{~mm}$, with a length-to-diameter ratio of 200 . This implies that the viscous scaled length of the wire $l^{+}$varied throughout the different cases. For all cases $l^{+}<25$, causing at worst a limited attenuation of the energy spectra for the small scales (Hutchins et al. 2009) which does not influence the results in the present paper. Wall-normal traverses are taken at multiple streamwise distances directly behind the jet by traversing from the wall upwards. The initial wall-normal position is determined using a digital microscope. The estimated uncertainty in this wall-normal position is $\epsilon_{y_{0}}<0.1 \mathrm{~mm}$. The probe is traversed using an encoder with a precision of an order of magnitude higher, implying that the uncertainty in wall-normal position is fully determined by the uncertainty of the initial position (i.e. $\epsilon_{y}<0.1 \mathrm{~mm}$ ).

The boundary-layer thickness $\delta$ and skin-friction velocity $U_{\tau}$ are determined from the unperturbed flow using the composite velocity profile fit of Chauhan et al. (2009). This fit is also used to obtain an accurate value for the offset in wall-normal position.

\subsection{Determining the jet velocity}

The mean blowing velocity in absence of the cross flow is used as the relevant jet velocity. For a sinusoidal signal the mean blowing velocity is given by $\bar{u}=u_{j, \max } / \pi$. The phase-averaged centreline velocity of the jet for a single case is presented for different distances to the jet in figure 2 . It can be seen that the maximum velocity occurs a small distance away from the orifice exit plane ( $y=3 \mathrm{~mm}$ for this specific case), which can 

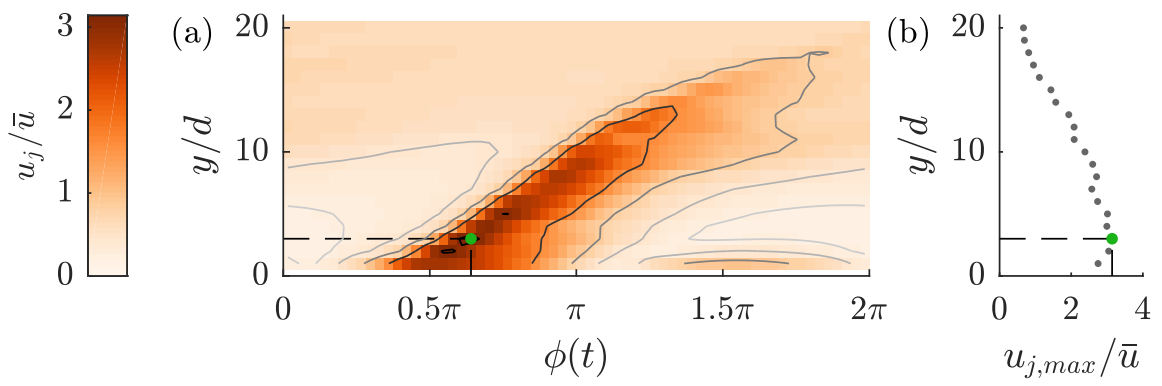

Figure 2. (a) Phase-locked jet-centreline velocity over 64 phases as function of wall-normal distance for case 1 as defined in table 1 (in absence of cross flow). (b) Variation of the maximum centreline velocity with wall-normal height. The wall-normal location and phase at which $u_{j, \max }$ occurs are indicated by the green dot.

be attributed to the formation of the vortex ring created by the jet (Smith and Glezer 1998). This true maximum velocity rather than the value at the orifice exit is used to calculate the mean blowing velocity. This figure also indicates the every weak effect of the suction phase which is located at $\phi=1.5 \pi$ and decays very quickly with distance from the orifice. Under the assumption that the suction phase has a negligible influence on the interaction with a cross flow a synthetic jet behaves as a pulsed jet.

\subsection{Determining the jet location}

Multiple definitions can be used to identify the location of a jet, including positions of local maxima in the velocity deficit, local vorticity maxima or the time-averaged streamline originating from the orifice (Mahesh 2013). Neither vorticity or streamlines can be determined from only the velocity magnitude. Identification of local maxima in the velocity deficit requires comparison of a perturbed to an unperturbed case, thereby increasing the uncertainty. Here we consider the location of the jet based on a single data set by decomposing the velocity signal as,

$$
U(y, t)=\langle U(y)\rangle+\widetilde{U}(y, \phi(t))+U^{\prime}(y, t),
$$

where $\langle U(y)\rangle$ is the time-averaged local velocity, $\widetilde{U}(y, \phi(t))$ is the phase-averaged local velocity at phase $\phi(t)$ and $U^{\prime}(y, t)$ are the local velocity fluctuations (similar to Hussain and Reynolds (1970)) these fluctuations include turbulence as well as fluctuations caused by the interaction of the jet with the cross flow which may occur at frequencies other than the jet frequency. A contour map of the phase-averaged component $\widetilde{U}$ as function of phase $\phi(t)$ and wall-normal location $y^{+}$is presented in figure 3a. A clear maximum of variations in phase can be identified at a wall-normal position indicated by the horizontal blue lines. The velocity at the phases indicated by the vertical lines is presented in figure $3 \mathrm{~b}$, where the root-mean-square value is indicated by the thick red lines. The location of the jet is defined as the location where this rms-value reaches its maximum. This location can be determined with an accuracy of one measurement point, leading to an estimated uncertainty on the wall-normal location of the jet of $\epsilon_{y}=0.17 y$, or $17 \%$, which is indicated by the blue error bar. This relatively large uncertainty is also displayed in the results in figure 4 and leads to a significant spread in the measurement points. When fitting the results using multiple points, this uncertainty decreases to acceptable levels as discussed below. 

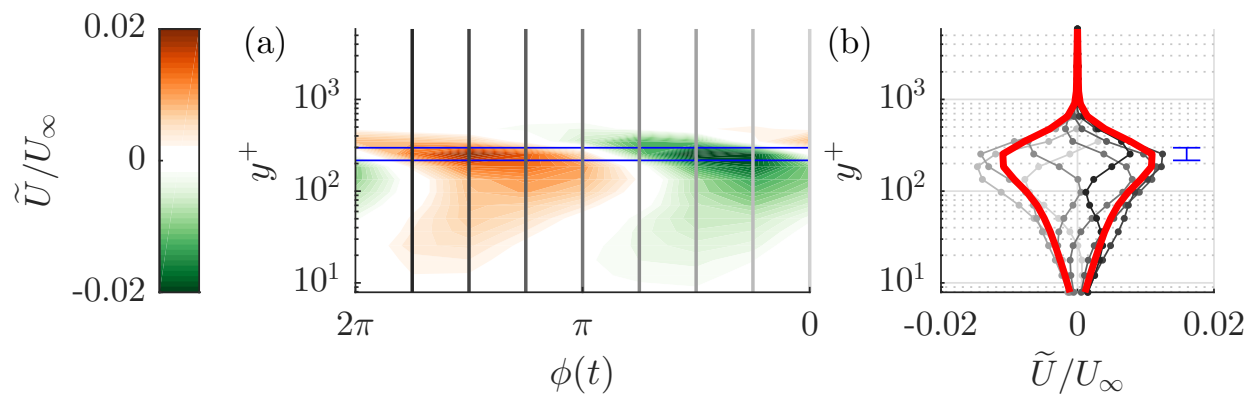

Figure 3. (a) Contour map of the phase-averaged local velocity $\widetilde{U}$ with wall-normal distance and phase. (b) Line plot of $\widetilde{U}$ where the line colour corresponds to colours of vertical lines in (a). The red lines indicate the root-mean-square over the eight phases, the blue lines indicate the location of the maximum.

\section{Results}

As discussed above, it is assumed that the trajectory of a synthetic jet issuing into a turbulent boundary layer scales with the Reynolds number $\left(R e_{\tau}\right)$, velocity ratio $(r)$ and some measure of frequency. The relevant non-dimensional group containing the jet frequency is unknown and could be any of the three groupings listed in equations 1.81.10. In an exploratory study these parameters are varied systematically to determine both the relevant normalisation of the frequency as well as the scaling of the trajectory as a function of this determined frequency, $R e_{\tau}$ and $r$. The analysis as presented here is highly dependent on the assumptions made for the important non-dimensional groups. The validity of these assumptions is investigated in appendix A where the same data is fitted against physical parameters first (i.e. $g(\ldots)=\bar{u}^{\alpha_{1}} f^{\alpha_{2}} D^{\alpha_{3}} U_{\infty}^{\alpha_{4}} \delta^{\alpha_{5}} \nu^{\alpha_{6}}$ ) after which non-dimensional groups are formed using the obtained coefficients. This leads to the same non-dimensional groups as those used in this section, indicating the validity of the assumed important non-dimensional groups. Using the results of the exploratory study, a validation study is performed in which the most important non-dimensional groups are varied systematically. All data points used in this study are provided in Appendix B.

\subsection{Exploratory study}

Jet- and cross-flow parameters are varied systematically as listed in table 1 . The first group of cases (1-4) considers a variation in $R e_{\tau}$ while keeping the velocity ratio constant and (roughly) matching the outer-scaled frequency $\left(f \delta / U_{\infty}\right)$ between cases. For the second group (5-8) the Reynolds number is varied for a constant blowing ratio while (roughly) matching the inner-scaled frequency $\left(f \nu / U_{\tau}^{2}\right)$. The third group (9-12) considers variations in blowing ratio while matching either inner- or outer-scaled frequencies or matching the blowing ratio and Reynolds number for a variation in frequency (cases 10 and 12). The passing frequency of the jet $\left(f D / U_{\infty}\right)$ is not matched systematically but varied/matched as a result of the others.

For each case the wall-normal location of the jet is determined at multiple positions downstream of the slot. The measured jet locations are presented in figure $4 \mathrm{a}$ with symbols corresponding to symbols in table 1. Error-bars indicate the uncertainty in determining the wall-normal location of the jet which is a result of the wall-normal spacing between measurement points. As expected the trajectories show some wallnormal variation with especially the cases of higher velocity ratio $(x, x$ and $\Delta)$ standing out. Cases containing three or more points are plotted with logarithmic axes in figure $4 \mathrm{~b}$. The points for each case are on a straight line, indicating that the trajectories follow a 
TABLe 1. Summary of case parameters for the exploratory study. Symbols indicate symbols used in figure 4 . The arbitrary value $D=1 \mathrm{~m}$ is used to quantify $f D / U_{\infty}$.

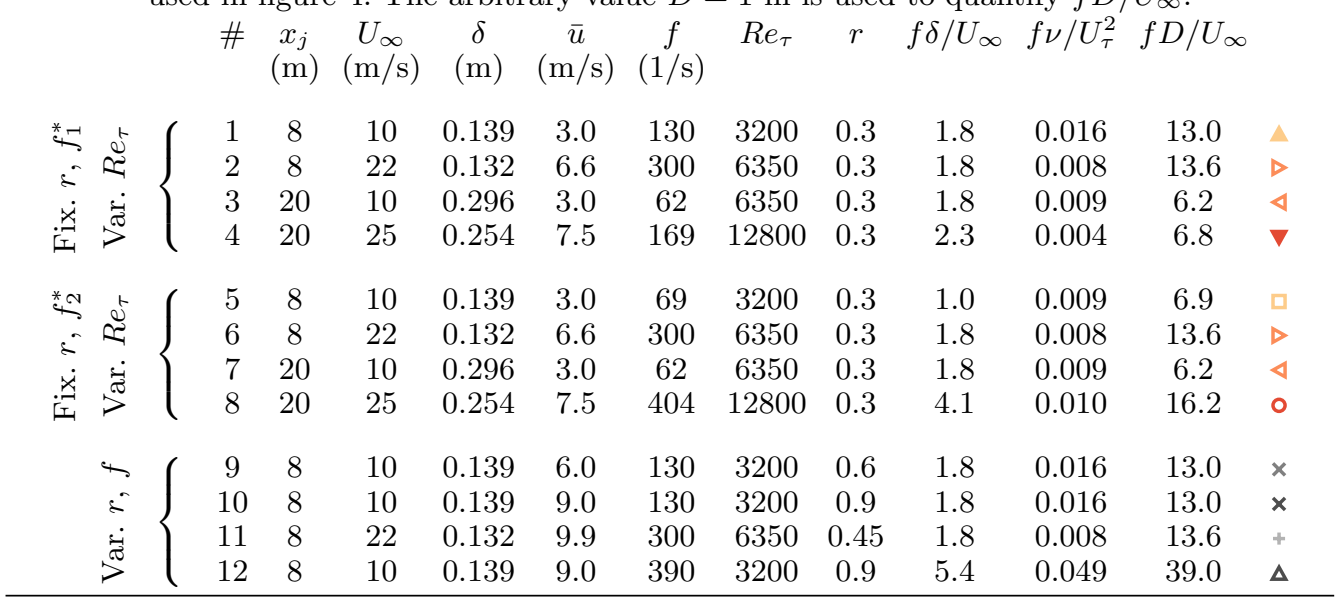

power law as hypothesised in $\S 1$. All cases in figure $4 \mathrm{~b}$ have the same slope, which suggest they can all be described using the same coefficient $n$ in equation 1.6.

The influence of the non-dimensional groups $\left(r, R e_{\tau}\right.$ and $\left.f^{*}\right)$ on the trajectory given by equation 1.6 is quantified by assuming $g(\ldots)=r^{c_{1}} R e_{\tau}^{c_{2}} f^{* c_{3}}$ (for $f_{1}^{*}, f_{2}^{*}$ and $f_{3}^{*}$ as defined in equations 1.8-1.10 independently). The coefficients $A, n$ and $c_{1-3}$ are fitted using nonlinear regression. The accuracy of each fit is measured as the root-mean-square of the residuals, i.e.

$$
\epsilon=\operatorname{rms}\left(y-A x^{n} g(\ldots)^{1-n}\right) .
$$

Fitting parameters and accuracy are listed in table 2. Fits are compared to an un-scaled power law $(g(\ldots)=1)$ and to scaling based on the velocity ratio only as in equation 1.2 $\left(g(\ldots)=r^{c}\right)$. Note that the value of $A$ depends on the choice of the orifice length-scale $D$ which is unknown. For each fit 28 data points are used, decreasing the estimated uncertainty in the wall-normal location (discussed in $\S 2.4$ ) to $\epsilon_{y}=0.17 y / \sqrt{28}=0.03 y$ or $3 \%$. For a nominal jet height of $y=0.02 \mathrm{~m}$ this uncertainty is $\epsilon_{y} \approx 0.6 \mathrm{~mm}$. Comparison of this uncertainty to the residuals of each fit as reported in table 2 suggests that the leading order uncertainty is in the fitting and not in the measurements.

Based on the residuals, proper normalisation of the frequency is as $f D / U_{\infty}(\epsilon=$ $1.6 \mathrm{~mm}$ ). The root-mean-square of the residuals using this scaling is almost twice as small as for the velocity-ratio scaling $(\epsilon=2.8 \mathrm{~mm})$ and almost four times as small as for the un-scaled case $(\epsilon=5.4 \mathrm{~mm})$. It should be noted that the residuals using the outerscaled frequency are only slightly higher (20\%) than when using the passing frequency. The difference between these normalisations is caused in this case (where $D$ is constant) by $\delta$ which is only varied by a factor two for the current experiments, explaining the small difference between the residuals.

The fit using the passing frequency shows a scaling of

$$
g(\ldots)=r^{1.26} R e_{\tau}^{-0.04}\left(f D / U_{\infty}\right)^{-0.56} .
$$

Measured trajectories are scaled using this expression in figures $4 \mathrm{~b}$ and $\mathrm{c}$, showing a reasonable collapse for all cases.

This result suggests that (I) the trajectory scales with $r^{1.26}$ which is equal to the 

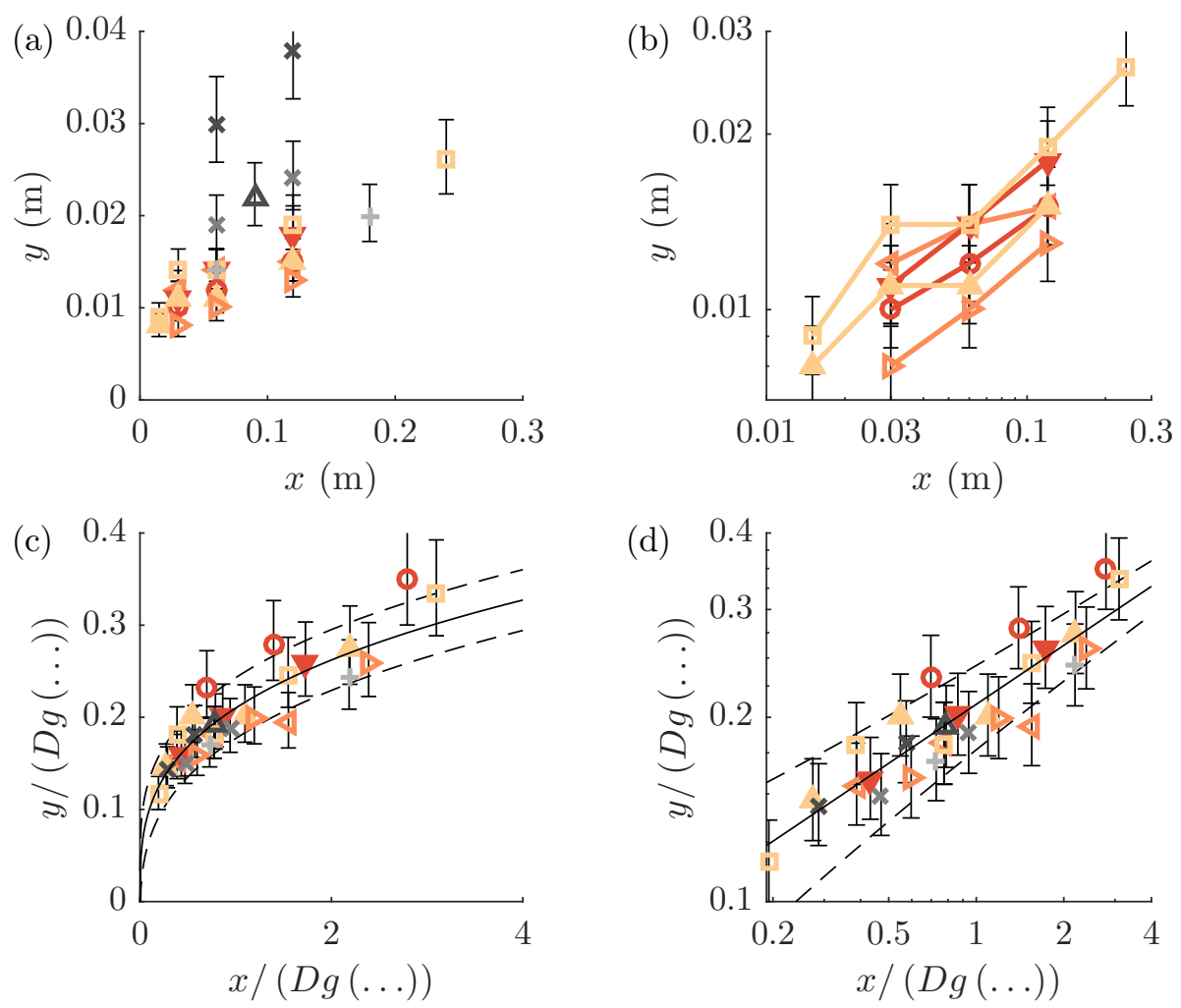

Figure 4. (a) Trajectories for all cases listed in table 1. (b) Trajectories of three data points and longer with logarithmic axes indicating a power-law behaviour. (c) Trajectories optimally scaled using the scaling factors in table 2. (d) Equal to (c) but with logarithmic axes. Solid lines indicate the fitted trajectory, dashed lines indicate the fit \pm the rms of the residuals. Error-bars indicate the error originating from the wall-normal spacing of measurement points as discussed in $\S 2.4$.

TABLE 2. Fitted coefficients and residuals for $y /(D g(\ldots))=A(x /(D g(\ldots)))^{n}$

$$
\begin{array}{ccccccc}
g\left(r^{c_{1}} \operatorname{Re}_{\tau}^{c_{2}} f^{* c_{3}}\right) & A & n & c_{1} & c_{2} & c_{3} & \epsilon(\mathrm{mm}) \\
1 & 0.04 & 0.38 & 0 & 0 & 0 & 5.4 \\
r^{c} & 0.07 & 0.34 & 0.95 & 0 & 0 & 2.8 \\
r^{c_{1}} R e_{\tau}^{c_{2}} f_{1}^{* c_{3}} & 0.04 & 0.30 & 1.15 & -0.53 & 0.13 & 1.9 \\
r^{c_{1}} R e_{\tau}^{c_{2}} f_{2}^{* c_{3}} & 0.30 & 0.32 & 0.91 & -0.20 & -0.37 & 2.4 \\
r^{c_{1}} R e_{\tau}^{c_{2}} f_{3}^{* c_{3}} & 0.26 & 0.32 & 1.26 & -0.04 & -0.56 & 1.6
\end{array}
$$

scaling for a steady jet, (II) even over the large range of Reynolds numbers tested here $\left(3200<R e_{\tau}<12800\right) R e_{\tau}$ only has a small effect on the trajectory and (III) scaling of the frequency of the jet is with the passing frequency of the flow over the orifice, $f D / U_{\infty}$.

\subsection{Validation study}

A second experiment is designed as validation of the leading terms $\left(r\right.$ and $\left.f D / U_{\infty}\right)$ in equation 3.2. In this experiment $R e_{\tau}$ is kept constant while $r$ and $f D / U_{\infty}$ are varied systematically as listed in table 3 .

Measured wall-normal locations of the jet for the validation study are presented in 
TABLE 3. Summary of case parameters for the validation study. Symbols indicate symbols used in figure 5 . The arbitrary value $D=1 \mathrm{~m}$ is used to quantify $f D / U_{\infty}$.

$\begin{array}{cccccccccc}\# & x_{j} & U_{\infty} & \delta & \bar{u} & f & R e_{\tau} & r & f D / U_{\infty} & \\ 13 & 20 & 10 & 0.296 & 4.5 & 62 & 6350 & 0.45 & 0.16 & \triangleleft \\ 14 & 20 & 10 & 0.296 & 3.0 & 140 & 6350 & 0.3 & 0.07 & \circ \\ 15 & 20 & 10 & 0.296 & 4.5 & 140 & 6350 & 0.45 & 0.07 & \circ \\ 16 & 20 & 10 & 0.296 & 6.75 & 140 & 6350 & 0.675 & 0.07 & \circ \\ 17 & 20 & 10 & 0.296 & 4.5 & 314 & 6350 & 0.45 & 0.03 & \square \\ 18 & 20 & 10 & 0.296 & 6.75 & 314 & 6350 & 0.675 & 0.03 & \square\end{array}$

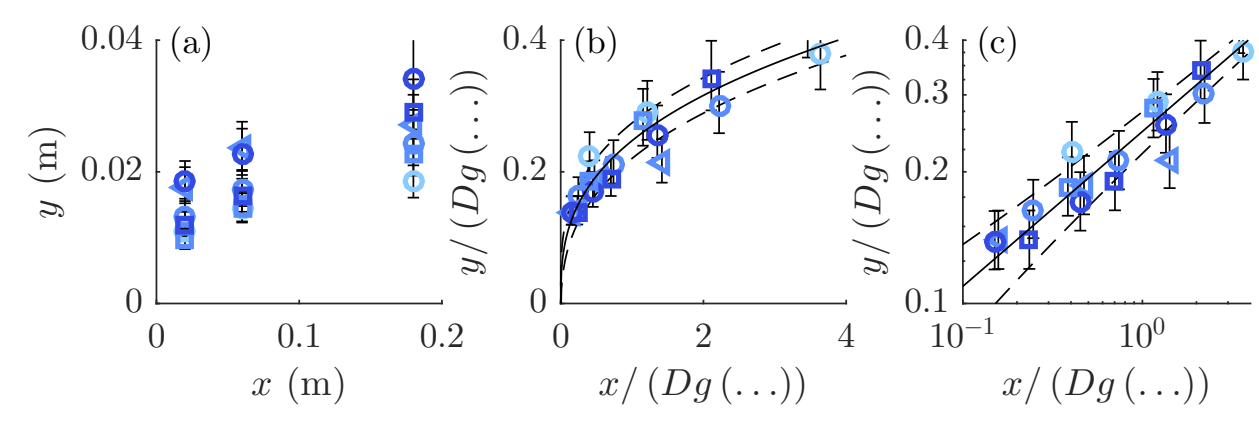

Figure 5. (a) Trajectories for all cases listed in table 3. (b,c) Trajectories scaled using equation 3.2. Solid lines indicate the fitted trajectory $(A=0.27, n=0.26)$, dashed lines indicate the fit \pm the rms of the residuals.

figure 5a. Fitted values for the power law are $A=0.27$ and $n=0.26$, which are slightly different from the values for the initial experiment $(A=0.26, n=0.32)$. Nevertheless, the normalized data in figures $5 \mathrm{~b}$ and c collapses onto a single power law (indicated by the black line), validating the scaling as listed in equation 3.2.

\subsection{Jet decay and very-far-field behaviour}

As discussed in $§ 2.4$ the location of the jet is determined using the cyclic velocity perturbations the jet creates in the cross-flow. These perturbations decay with streamwise distance form the jet exit and the streamwise extent of the measurements as presented above is limited by this decay. For the most-downstream points of each case the rootmean-square of the velocity fluctuations has typically decayed down to $0.1 \%$ of the freestream velocity and the jet could not be detected further downstream.

This same decay implies that in the very-far-field, where coherent structures created by the jet have vanished, the fluid ejected by the jet will not penetrate the flow further and the trajectory becomes horizontal. This may suggest a deviation from the power law (which does not become fully horizontal) in the very-far-field.

\section{Discussion}

The derived scaling for the trajectory of a synthetic jet issuing into a turbulent boundary layer as stated in equation 3.2 depends on three non-dimensional groups.

Analogous to steady jets, the trajectory scales with the velocity ratio as $y / r^{c} \propto\left(x / r^{c}\right)^{n}$. As discussed above, for steady jets usually a value of $c=1$ is stated, but in general $0<c<2$. The value found for synthetic jets, $c=1.26$, is well within this range, showing analogy in the effect of the velocity ratio on trajectories of steady jets and synthetic jets. 
For the investigated range of parameters $\left(3200<R e_{\tau}<12800\right)$, the friction Reynolds number is found to only have a small influence on the trajectory of the jet, scaling as $y / R e_{\tau}^{-0.04} \propto\left(x / R e_{\tau}^{-0.04}\right)^{n}$. This coefficient may be considered to be within experimental uncertainty. Therefore, the trajectory of the jet in the cross flow is at best weakly dependent on the Reynolds number of the boundary layer and is most likely to be independent of it. This suggests that further studies can be performed in other facilities which do not have the capability of reaching high friction Reynolds numbers. It should be noted that $U_{\tau}$ may have an additional influence on the trajectory which could not be independently measured due to the dependence of $c_{f}$ on $R e_{\tau}$. The independence of the trajectory on $R e_{\tau}$ does not necessarily imply that the trajectory is equal in laminar boundary layers. Due to the fuller velocity profile of (high-Reynolds number) turbulent boundary layers, most of the circulation is concentrated relatively close to the wall compared to laminar boundary layers. As discussed above, this circulation interacts with the vortical structure formed by the jet, leading to geometrically complicated vortical structures. This suggests that turbulent boundary layers have a much larger effect on the created vortical structures and therewith a different effect on the trajectory. It should be noted that the stated independence of Reynolds number is based on high Reynolds numbers $\left(R e_{\tau}>3200\right)$ and its scaling is unknown when the laminar limit is approached at low Reynolds numbers. When the boundary-layer thickness is considered independently (see appendix A) it influences the scaling parameter as $g(\ldots) \propto \delta^{-0.08}$. While the boundary-layer thickness is varied by a factor 2.2 in the present study, it only altered the scaling parameter by $6 \%$. Therefore, other non-dimensional groups containing $\delta$ ( such as $\delta / D)$ are considered negligible.

It is found that the trajectory scales with the term $f D / U_{\infty}$, where $D$ is a representative slot dimension added from dimensional arguments. The exact nature of $D$ can only be determined by varying the slot dimensions, which was outside the scope of the parametric study in the performed experiments, but it is assumed that $D$ is a function of both the slot width $d$ and length $l$. The term $f D / U_{\infty}$ represents a ratio between two length scales, where $U_{\infty} / f$ is related to the separation distance between subsequent jet pulses created at time interval $1 / f$ and with streamwise advection velocity $\propto U_{\infty}$. These length scales are schematically represented in figure $6 \mathrm{a}$, where it is assumed that the size of jet pulses/vortical structures depends on the representative size of the orifice $D$. As presented in figure $6 \mathrm{~b}$, when increasing this ratio an interesting phenomenon occurs near unity where the pulses of the jet form a seemingly continuous jet. At the same time when $f D / U_{\infty}=1$ (and neglecting the influence of $R e_{\tau}^{-0.04}$ ) the scaling factor in equation 3.2 is given by $g(\ldots)=r^{1.26}$, recovering the scaling for a steady jet (as in equation 1.2).

As discussed by Shapiro et al. (2006) and Karagozian (2014), the wave-form used to actuate an unsteady jet has a large influence on the trajectory. Therefore, results of pulsed and forced jets (often actuated using a square-wave) may differ from the results obtained in the present study. However, following the above discussion and the schematic in figure 6 , it may be assumed that the obtained scaling holds for unsteady jets that create a train of distinct vortical structures, which (under certain conditions) has been observed for both pulsed jets (e.g. Jabbal and Zhong 2008) and forced jets (e.g. Johari 2006).

\section{Conclusions}

Scaling of the trajectory of a synthetic jet issuing into a turbulent boundary layer is derived. This problem can be described by the slot length $l$ and width $d$, the jet frequency $f$ and mean blowing velocity $\bar{u}$, the free-stream velocity $U_{\infty}$, the boundarylayer thickness $\delta$, the friction velocity $U_{\tau}$ and the kinematic viscosity $\nu$. Six independent 
(a)

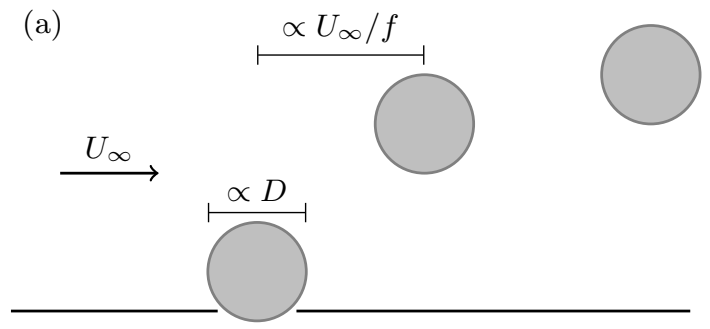

(b)

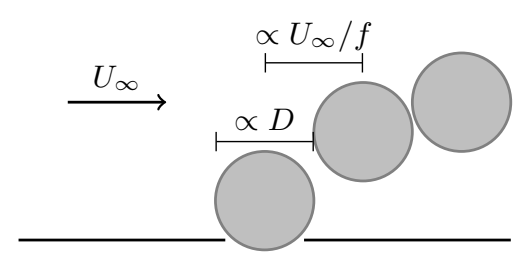

FiguRE 6. Schematic indicating the physical meaning of the non-dimensional group $f D / U_{\infty}$ which is considered as the ratio between length scales $U_{\infty} / f$, representing the streamwise distance between subsequent pulses of the jet and $D$, representing the size of these pulses. (a) A case with $f D / U_{\infty}<<1$ and (b) the case $f D / U_{\infty}=1$, showing an almost continuous jet.

non-dimensional groups can be formed out of these parameters. However, due to the nature of the experiment the aspect ratio of the jet $A R=l / d$ as well as the ratio $U_{\infty} / U_{\tau}$ are fixed, leaving four independent non-dimensional groups to describe scaling of the trajectory of the jet. Fitting of trajectories for a wide parametric study showed that only three independent non-dimensional groups are required to describe these trajectories. The trajectories are shown to scale as

$$
\frac{y / D}{r^{1.26} R e_{\tau}^{-0.04}\left(f D / U_{\infty}\right)^{-0.56}}=A\left(\frac{x / D}{r^{1.26} R e_{\tau}^{-0.04}\left(f D / U_{\infty}\right)^{-0.56}}\right)^{n},
$$

where the scaling factors in the denominator are given by:

(i) $r^{1.26}$, showing analogy to trajectories of steady jet in cross flow which scales with $r^{c}$ for typical values of $0<c<2$.

(ii) $R e_{\tau}^{-0.04}$, indicating only a weak dependence on the friction Reynolds number. This enables future studies in facilities with lower Reynolds numbers.

(iii) $\left(f D / U_{\infty}\right)^{-0.56}$, where $D$ is an unknown combination of the slot dimensions $l$ and $d$. This term indicates the relevant non-dimensional number for the jet frequency. This group gives the ratio between two length scales; $U_{\infty} / f$, relating to the streamwise spacing between subsequent pulses and $D$, relating to the size of these pulses. It can be argued that when the separation between pulses equals the pulse size a continuous jet is formed. At the same time, when this ratio goes to unity the scaling for the trajectory of a steady jet in cross flow is recovered. These arguments hold for other types of unsteady jets such as pulsed and forced jets. It may be assumed that (under certain conditions) the scaling with frequency is equal for synthetic, pulsed and forced jets. The nature of the relevant orifice dimension $D$ and the relation to the pulse size as hypothesised above are subjects for further study. Assuming that a single orifice length scale $D$ exists, results are expected to hold for other orifice shapes when normalized by their respective length scales.

The study was motivated by the application in skin-friction control, which is reflected in the shape of the jet orifice (rectangular with the long dimension aligned with the flow) and the low velocity ratios used in the study. One distinctive aspect of the study are the high Reynolds numbers of the turbulent boundary layers. This is the first study known to the authors to investigate the effect of the friction Reynolds number on the trajectory of a (synthetic) jet in a cross flow. Furthermore, this is the first study known to the authors to systematically study the effect of the frequency of an unsteady jet on the trajectory. 
TABLE 4. Fitted coefficients and residuals for equation A 1

$\begin{array}{lllllllll}A & n & \alpha_{1} & \alpha_{2} & \alpha_{3} & \alpha_{4} & \alpha_{5} & \alpha_{6} & \epsilon(\mathrm{mm})\end{array}$

$\begin{array}{lllllllll}0.21 & 0.32 & 1.22 & -0.55 & -0.55 & -0.75 & -0.08 & 0.08 & 1.5\end{array}$

TB and BG acknowledge the financial support from the European Research Council (ERC grant agreement no. 277472), Engineering and Physical Sciences Research Council (EPSRC grant no. EP/L006383/1) and Airbus. IM and NH acknowledge the support of the Australian Research Council.

\section{Appendix A. Fitting of dimensional parameters}

Fitting the scaling parameter $g(\ldots)$ against pre-assumed non-dimensional groups as in equation 1.11 limits the result to these assumed groups. The purpose of this appendix is to overcome this limit by fitting the scaling parameter against dimensional parameters. As listed in $\S 1.5$, the parameters describing the interaction of a synthetic jet with a cross flow are the velocity magnitude $\bar{u}$, frequency $f$ and (unknown) orifice length-scale $D$ of the jet as well as the free-stream velocity $U_{\infty}$, boundary-layer thickness $\delta$, friction velocity $U_{\tau}$ and kinematic viscosity $\nu$ of the cross flow. The friction velocity is related to the free-stream velocity through the skin-friction coefficient, $c_{f}=2 U_{\tau}^{2} / U_{\infty}^{2}$, which is a function of $\log \left(R e_{x}\right)$. For the values of $R e_{x}$ used in the present study the ratio between the friction velocity and free-stream velocity is fairly constant (i.e. $U_{\infty} \approx U_{\tau} \beta$ for a constant $\beta$ ). This means that they cannot be fitted independently and $U_{\tau}$ is left out of consideration for the fit. This leads to a description of the trajectory of

$$
\frac{y}{\left(\bar{u}^{\alpha_{1}} f^{\alpha_{2}} D^{\alpha_{3}} U_{\infty}^{\alpha_{4}} \delta^{\alpha_{5}} \nu^{\alpha_{6}}\right) D}=A\left(\frac{x}{\left(\bar{u}^{\alpha_{1}} f^{\alpha_{2}} D^{\alpha_{3}} U_{\infty}^{\alpha_{4}} \delta^{\alpha_{5}} \nu^{\alpha_{6}}\right) D}\right)^{n} .
$$

As discussed in the main text, the orifice length-scale $D$ is unknown but constant and fitted as $D=1 \mathrm{~m}$, which will influence the value of coefficient $A$ only. The variation in the viscosity $\nu$ throughout the experiments was negligible and $\alpha_{6}$ can not be reliably fitted by itself. Coefficients $\alpha_{3}$ and $\alpha_{6}$ can then be used for dimensional reasons since the dimensions of the terms within the brackets in the denominator of equation A 1 are

$$
[g(\ldots)]=\mathrm{m}^{\alpha_{1}+\alpha_{3}+\alpha_{4}+\alpha_{5}+2 \alpha_{6}} \mathrm{~s}^{-\alpha_{1}-\alpha_{2}-\alpha_{4}-\alpha_{6}} .
$$

Given that this term is dimensionless, $\alpha_{3}$ and $\alpha_{6}$ can be determined as

$$
\begin{aligned}
& \alpha_{3}=\alpha_{1}+2 \alpha_{2}+\alpha_{4}-\alpha_{5}, \\
& \alpha_{6}=-\alpha_{1}-\alpha_{2}-\alpha_{4} .
\end{aligned}
$$

The coefficients $A, n$ and $\alpha_{1-6}$ are fitted against the data presented in $\S 3.1$ using nonlinear regression. Fitted coefficients and the root-mean-square of the residuals $(\epsilon)$ are presented in table 4 . In dimensional parameters, the scaling parameter is given by

$$
g(\ldots)=\bar{u}^{1.22} f^{-0.55} D^{-0.55} U_{\infty}^{-0.75} \delta^{-0.08} \nu^{0.08} .
$$

Following Buckingham-Pi theorem, four independent non-dimensional groups can be formed out of these six dimensional parameters. It should be noted that grouping these parameters can be done in numerous different ways and the chosen groups depend on assumptions.

Following the literature it seems sensible to group the jet velocity and cross-flow 
velocity into a velocity ratio $r^{1.22}=\left(\bar{u} / U_{\infty}\right)^{1.22}$. From the equal coefficients for the boundary-layer thickness and viscosity, it seems sensible to group these into the friction Reynolds number $R e_{\tau}^{-0.08}=\left(\delta U_{\tau} / \nu\right)^{-0.08}$, where the direct relationship between the friction velocity and free-stream velocity is used to substitute $U_{\infty}^{0.08}=U_{\tau}^{0.08} \beta^{0.08}$ for $\beta$ a constant that will alter coefficient $A$. Using these choices, the scaling parameter has reduced to

$$
g(\ldots)=r^{1.22} R e_{\tau}^{-0.08} f^{-0.55} D^{-0.55} U_{\infty}^{0.55},
$$

which indicates a non-dimensional frequency $\left(f D / U_{\infty}\right)^{-0.55}$. Note that this has led to only three non-dimensional groups where four independent groups were possible. Also note that this analysis has obtained the same non-dimensional groups as the analysis in $\S 3$ with the difference in coefficients being within experimental error.

Using different assumptions, a variety of different groupings are possible. Most notably, the scaling parameter can be represented as

$$
g(\ldots)=r^{0.67}(L / D)^{0.55} \operatorname{Re}_{\tau}^{-0.08},
$$

where $L / D$ is the non-dimensional stroke length which is an important parameter for the formation of vortex rings. This parameter was left out of the assumptions in $\S 1.5$ under the assumption that the scaling of the frequency should include cross-flow parameters. It is included here because it shows some similarity with a theoretical scaling for the trajectory of isolated vortex rings as derived by Johari (2006). This scaling can be represented as

$$
g(\ldots)=r^{0.33}(L / D)^{0.33} .
$$

Although these expressions take the same form, the coefficients are somewhat different. When this scaling in equation A 8 is used to normalize the data (using a best-fit for $A$ and $n$ ), a root-mean-square of the residuals of $\epsilon=3.1 \mathrm{~mm}$ is obtained. This deviation is larger than when using just velocity-ratio scaling (see table 2), invalidating this scaling for the data in the present study.

Out of all the possible groupings of physical parameters in equation A 5 the authors deem the one discussed above and presented in equation A 6 most feasible, substantiating the scaling derived in the study.

\section{Appendix B. Data set used in this study}

The full set of data used in this study, as plotted in Figures 4 and 5 is provided in Table 5. Using these (dimensional) data points and the parameters provided in Tables 1 and 3, all plots in Figures 4 and 5, as well as the discussed fits can be reproduced.

\section{REFERENCES}

M. R. Abbassi, W. Baars, N. Hutchins, and I. Marusic. Skin-friction drag reduction in a highReynolds-number turbulent boundary layer via real-time control of large-scale structures. Int. J. Heat Fluid Flow, 67(B):30-41, 2017.

J. Broadwell and R. Breidenthal. Structure and mixing of a transverse jet in incompressible flow. J. Fluid Mech., 148:405-412, 1984.

J. E. Cater and J. Soria. The evolution of round zero-net-mass-flux jets. J. Fluid Mech., 472: 167-200, 2002.

K. A. Chauhan, P. A. Monkewitz, and H. M. Nagib. Criteria for assessing experiments in zero pressure gradient boundary layers. Fluid Dyn. Res., 41(021404):1-23, 2009.

J. Dandois, E. Garnier, and P. Sagaut. Numerical simulation of active separation control by a synthetic jet. J. Fluid Mech., 574:25-58, 2007. 
$\begin{array}{ccccccccccc}\text { TABle } & \text { 5. Data points used in this study as plotted in Figures } 4 \text { and } 5 . \\ & x(\mathrm{~m}) & 0.015 & 0.02 & 0.03 & 0.06 & 0.09 & 0.12 & 0.18 & 0.24 \\ \# & y(\mathrm{~m}) & & & & & & & & \\ 1 & & 0.008 & - & 0.011 & 0.011 & - & 0.015 & - & - \\ 2 / 6 & - & - & 0.008 & 0.010 & - & 0.013 & - & - \\ 3 / 7 & - & - & 0.012 & 0.014 & - & 0.015 & - & - \\ 4 & - & - & 0.011 & 0.014 & - & 0.018 & - & - \\ 5 & 0.009 & - & 0.014 & 0.014 & - & 0.019 & - & 0.026 \\ 8 & - & - & 0.010 & 0.012 & - & 0.015 & - & - \\ 9 & - & - & - & 0.019 & - & 0.024 & - & - \\ 10 & - & - & - & 0.030 & - & 0.038 & - & - \\ 11 & - & - & - & 0.014 & - & - & 0.020 & - \\ 12 & - & - & - & - & 0.022 & - & - & - \\ 13 & - & 0.018 & - & 0.024 & - & - & 0.027 & - \\ 14 & - & 0.011 & - & 0.014 & - & - & 0.019 & - \\ 15 & - & 0.013 & - & 0.017 & - & - & 0.024 & - \\ 16 & - & 0.019 & - & 0.023 & - & - & 0.034 & - \\ 17 & - & 0.010 & - & 0.015 & - & - & 0.023 & - \\ 18 & - & 0.012 & - & 0.016 & - & - & 0.029 & -\end{array}$

J. Davitian, C. Hendrickson, R. T. M'Closkey, and A. R. Karagozian. Strategic control of transverse jet shear layer instabilities. AIAA J., 48(9):2145-2156, 2010.

A. Eroglu and R. E. Breidenthal. Structure, penetration, and mixing of pulsed jets in crossflow. AIAA J., 39(3):417-423, 2001.

T. Fric and A. Roshko. Vortical structure in the wake of a transverse jet. J. Fluid Mech., 279: 1-47, 1994.

M. Gharib, E. Rambod, and K. Shariff. A universal time scale for vortex ring formation. J. Fluid Mech., 360:121-140, 1998.

A. Hussain and W. Reynolds. The mechanics of an organized wave in turbulent shear flow. $J$. Fluid Mech., 41:241-258, 1970.

N. Hutchins, T. B. Nickels, I. Marusic, and M. S. Chong. Hot-wire spatial resolution issues in wall-bounded turbulence. J. Fluid Mech., 635:103-136, Sep 2009.

M. Jabbal and S. Zhong. The near wall effect of synthetic jets in a boundary layer. Int. J. Heat Fluid Flow, 29(1):119-130, 2008.

M. Jabbal and S. Zhong. Particle image velocimetry measurements of the interaction of synthetic jets with a zero-pressure gradient laminar boundary layer. Phys. Fluids, 22(6):063603, 2010. .

H. Johari. Scaling of fully pulsed jets in crossflow. AIAA J., 44(11):2719-2725, 2006.

H. Johari, M. Pacheco-Tougas, and J. C. Hermanson. Penetration and mixing of fully modulated turbulent jets in crossflow. AIAA J., 37(7):842-850, 1999.

A. R. Karagozian. An analytical model for the vorticity associated with a transverse jet. AIAA J., 24(3):429-436, 1986.

A. R. Karagozian. The jet in crossflow. Phys. Fluids, 26:101303, 2014.

J. F. Keffer and W. D. Baines. The round turbulent jet in a cross-wind. J. Fluid Mech., 15(4): 481-496, 1962.

W. Kim, C. Kim, and K. J. Jung. Separation control characteristics of synthetic jets depending on exit configuration. AIAA J., 50(3):559-570, 2012.

J. Klewicki. Reynolds number dependence, scaling, and dynamics of turbulent boundary layers. J. Fluids Eng., 132(9):094001, 2010.

T. T. Lim, K. B. Lua, and K. Thet. Does kutta lift exist on a vortex ring in a uniform cross flow? Phys. Fluids, 20(5):051701, 2008.

K. Mahesh. The interaction of jets with crossflow. Ann. Rev. Fluid Mech., 45(1):379-407, Jan 2013. .

R. J. Margason. Fifty years of jet in cross flow research. In AGARD, Computational and 
Experimental Assessment of Jets in Cross Flow, pages AGARD-CP-534. Advis. Group Aeronaut. Res. Dev., Washington, DC, 1993.

I. Marusic, R. Mathis, and N. Hutchins. Predictive model for wall-bounded turbulent flow. Science, 329(5988):193-196, 2010.

R. T. M'Closkey, J. M. King, L. Cortelezzi, and A. R. Karagozian. The actively controlled jet in crossflow. J. Fluid Mech., 452:325-335, Feb 2002. .

S. Megerian, J. Davitian, B. Alves, and A. R. Karagozian. Transverse-jet shear-layer instabilities. part 1. experimental studies. J. Fluid Mech., 593:93-129, 2007.

D. N. Miller, P. J. Yagle, E. E. Bender, and B. R. Smith. A computational investigation of pulsed injection into a confined expanding crossflow. In 15th AIAA Computational Fluid Dynamics Conference, pages 2001-3026, Anaheim, CA, USA, 2001.

F. Muldoon and S. Acharya. Dns study of pulsed film cooling for enhanced cooling effectiveness. Int. J. Heat and Mass Transf., 52:3118-3127, 2009.

S. Muppidi and K. Mahesh. Study of trajectories of jets in crossflow using direct numerical simulations. J. Fluid Mech., 530:81-100, 2005.

S. Narayanan, P. Barooah, and J. M. Cohen. Dynamics and control of an isolated jet in crossflow. AIAA J., 41(12):2316-2330, 2003.

T. B. Nickels, I. Marusic, S. Hafez, and M. S. Chong. Evidence of the $k_{1}^{-1}$ law in a highreynolds-number turbulent boundary layer. Phys. Rev. Lett., 95(7):074501, Aug 2005.

C. O'Farrell and J. O. Dabiri. Pinch-off of non-axisymmetric vortex rings. J. Fluid Mech., 740: 61-96, 2014. .

R. Rathnasingham and K. S. Breuer. Active control of turbulent boundary layers. J. Fluid Mech., 495:209-233, 2003. .

R. Sau and K. Mahesh. Dynamics and mixing of vortex rings in crossflow. J. Fluid Mech., 604: 389-409, May 2008. .

S. R. Shapiro, J. M. King, R. T. M'Closkey, and A. R. Karagozian. Optimization of controlled jets in crossflow. AIAA J., 44(6):1292-1298, 2006.

J. Shuster, R. Pink, D. McEligot, and D. Smith. The interaction of a circular synthetic jet with a cross-flow boundary layer. In 35th AIAA Fluid Dynamics Conference and Exhibit, Toronto, ON, Canada, Jun 2005. .

B. L. Smith and A. Glezer. The formation and evolution of synthetic jets. Phys. Fluids, 10(9): 2281-2297, 1998. .

D. R. Smith. Interaction of a synthetic jet with a crossflow boundary layer. AIAA J., 40(11): 2277-2288, 2002. .

S. H. Smith and M. G. Mungal. Mixing, structure and scaling of the jet in crossflow. J. Fluid Mech., 357:83-122, 1998.

A. J. Smits, B. J. McKeon, and I. Marusic. High reynolds number wall turbulence. Annu. Rev. Fluid Mech., 43(1):353-375, 2011.

T. Van Buren and M. Amitay. Comparison between finite-span steady and synthetic jets issued into a quiescent fluid. Exp. Therm. Fluid Sci., 75:16-24, 2016.

T. Van Buren, E. Whalen, and M. Amitay. Vortex formation of a finite-span synthetic jet: effect of rectangular orifice geometry. J. Fluid Mech., 745:180-207, 2014. .

T. Van Buren, C. M. Leong, E. Whalen, and M. Amitay. Impact of orifice orientation on a finite-span synthetic jet interaction with a crossflow. Phys. Fluids, 28:037106, 2016.

P. J. Vermeulen, C. Chin, and W. K. Yu. Mixing of an acoustically pulsed air jet with a confined crossflow. J. Propulsion, 6(6):777-783, 1990.

J. M. Wu, A. D. Vakili, and F. M. Yu. Investigation of the interacting flow of nonsymmetric jets in crossflow. AIAA J., 26(8):940-947, 1988. 